\title{
Reduced risk of hospitalisation among reported COVID-19 cases infected with the SARS-CoV-2 Omicron BA.1 variant compared with the Delta variant, Norway, December 2021 to January 2022
}

\author{
Lamprini Veneti ${ }^{1}$, Håkon Bøås ${ }^{2}$, Anja Bråthen Kristoffersen ${ }^{3}$, Jeanette Stålcrantz ${ }^{2,4}$, Karoline Bragstad ${ }^{5}$, Olav Hungnes ${ }^{5}$, \\ Margrethe Larsdatter Storm ${ }^{6}$, Nina Aasand ${ }^{6}$, Gunnar Rø ${ }^{3}$, Jostein Starrfelt' ${ }^{1}$, Elina Seppälä ${ }^{2}$, Reidar Kvåle ${ }^{7,8}$, Line Vold ${ }^{1}$, Karin \\ Nygård ${ }^{1}$, Eirik Alnes Buanes, ${ }^{7,9}$, Robert Whittaker ${ }^{2}$ \\ 1. Department of Infection Control and Preparedness, Norwegian Institute of Public Health, Oslo, Norway \\ 2. Department of Infection Control and Vaccines, Norwegian Institute of Public Health, Oslo, Norway \\ 3. Department of Method Development and Analytics, Norwegian Institute of Public Health, Oslo, Norway \\ 4. European Program for Intervention Epidemiology Training, European Centre for Disease Prevention and Control, Stockholm, \\ Sweden \\ 5. Department of Virology, Norwegian Institute of Public Health, Oslo, Norway \\ 6. Department of Infectious Disease Registries, Norwegian Institute of Public Health, Oslo, Norway \\ 7. Department of Anaesthesia and Intensive Care, Haukeland University Hospital, Bergen, Norway \\ 8. Department of Clinical Medicine, University of Bergen, Bergen, Norway \\ 9. Norwegian Intensive Care and Pandemic Registry, Haukeland University Hospital, Bergen, Norway
}

Correspondence: Lamprini Veneti (lamprini.veneti@fhi.no)

Citation style for this article:

Veneti Lamprini, Bøås Håkon, Bråthen Kristoffersen Anja, Stålcrantz Jeanette, Bragstad Karoline, Hungnes Olav, Storm Margrethe Larsdatter, Aasand Nina, Rø Gunnar, Starrfelt Jostein, Seppälä Elina, Kvåle Reidar, Vold Line, Nygård Karin, Buanes Eirik Alnes, Whittaker Robert. Reduced risk of hospitalisation among reported COVID-19 cases infected with the SARS-CoV-2 Omicron BA.1 variant compared with the Delta variant, Norway, December 2021 to January 2022 . Euro Surveill. 2022;27(4):pii=2200077. https://doi.org/10.2807/1560-7917.ES.2022.27.4.2200077

We included 39,524 COVID-19 Omicron and 51,481 Delta cases reported in Norway from December 2021 to January 2022 . We estimated a $73 \%$ reduced risk of hospitalisation (adjusted hazard ratio: 0.27; $95 \%$ confidence interval: $0.20-0.36)$ for Omicron compared with Delta. Compared with unvaccinated groups, Omicron cases who had completed primary two-dose vaccination 7-179 days before diagnosis had a lower reduced risk than Delta (66\% vs $93 \%$ ). People vaccinated with three doses had a similar risk reduction ( $86 \%$ vs $88 \%$ ).

In Norway, the first coronavirus disease (COVID-19) cases infected with the severe acute respiratory syndrome coronavirus 2 (SARS-CoV-2) variant Omicron (Phylogenetic Assignment of Named Global Outbreak Lineages (Pangolin) designation B.1.1.529) substrain BA.1 were detected in an outbreak following a social gathering on 26 November 2021 where the suspected index case had reported recent travel to South Africa [1]. Since then, testing activity has been high $[2,3]$, and the proportion of cases screened for variant weekly ranged from 49 to $67 \%$ up to early January 2022. Omicron became the dominant circulating variant in late December 2021 (Figure 1).

We used linked individual-level data from the Norwegian Preparedness Registry (Beredt C19) [4] to estimate the risk of hospitalisation for reported Omicron cases compared with Delta (B.1.617.2) cases. We also compared the length of hospital stay (LoS) and estimated the risk of admission to an intensive care unit (ICU) among hospitalised Omicron and Delta cases, and described deaths in the study cohort.

\section{Study population}

We analysed cases of COVID-19 with a date of positive test between 6 December 2021 and 9 January 2022. We extracted data from Beredt $\mathrm{C}_{19}$ on 20 January 2022, allowing 10 days of follow-up since last positive test. Further details on the data sources and definitions can be found in the Supplement, section 1. Of 158,561 cases reported in the study period, 155,388 (98\%) had a national identity number. The national identity number was essential to link data from all registries used in the analysis. Of these 155,388, 91,772 (59\%) were screened for variants. Variants were identified based on whole genome sequencing, Sanger partial S-gene sequencing or PCR screening targeting specific single nucleotide polymorphisms, insertions or deletions that reliably differentiate between Omicron and other variants. During the study period, $11 \%$ of variants were sequenced.

We assessed the representativeness of the 91,772 screened cases among the 155,388 reported cases. The proportion of cases screened among hospitalised cases was higher than among non-hospitalised cases (73\% vs 59\%). We expect this difference to have a 
Proportion of reported COVID-19 cases with data on virus variant, and proportion of Delta and Omicron by week of sampling, Norway, 1 November 2021-9 January 2022 ( $\mathrm{n}=239,391)$

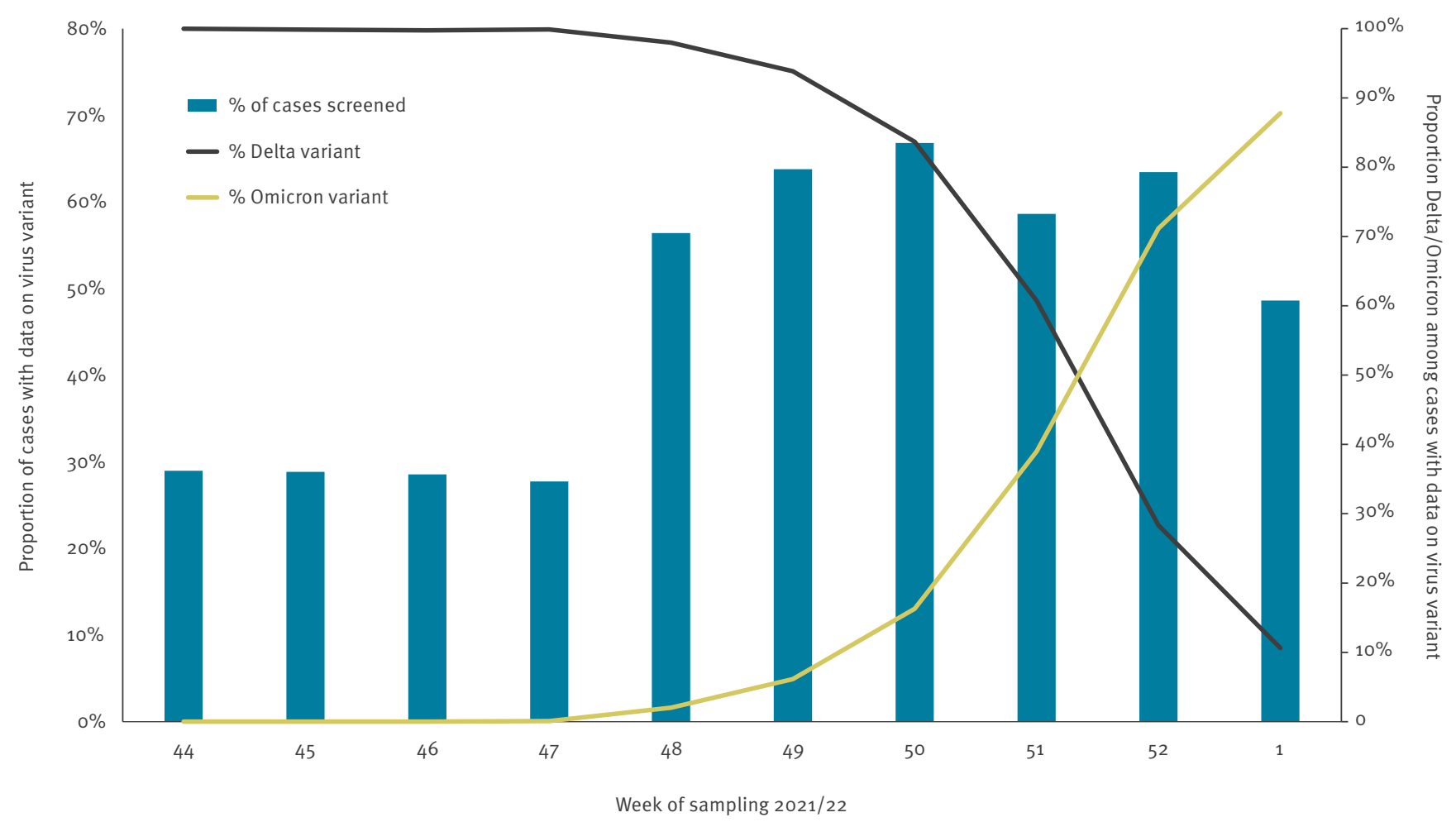

COVID-19: coronavirus disease.

Testing activity and screening of cases was enhanced after the detection of the first Omicron (B.1.1.529) cases in Norway (week 48). Variants other than Delta (B.1.617.2) and Omicron were infrequently detected during the study period (week 49-1). During the study period, 155,388 cases were reported and 91,772 (59\%) were screened for variants. Of those $91,772,55 \%$ were Delta, $44 \%$ Omicron BA.1 and $0.3 \%$ Omicron BA.2 ( $n=242$ in week 1$)$.

limited impact on our estimates, given the small proportion of all cases admitted to hospital, and that screening of hospitalised cases did not depend on variant exposure. Section 2 of the Supplement provides a detailed comparison of screened and not-screened cases.

We only included cases infected with SARS-CoV-2 variants Omicron (excluding all 242 sub-variant BA.2 cases that were reported in week 1) and Delta. Our main outcome was hospitalisation $\leq 2$ days before and $\leq 28$ days following a positive COVID-19 test, where COVID-19 was the reported main cause of admission. To avoid bias, we excluded 301 Omicron and Delta cases hospitalised with another or unknown main cause of admission.

Overall, 39,524 Omicron (43\%) and 51,481 (57\%) Delta cases were included. See Table 1 for characteristics of our retrospective study cohort. The median time since last vaccination dose was 201 days (interquartile range (IQR): 190-216; maximum: 316) for cases who had completed primary vaccination with maximum two doses $\geq 180$ days before positive test, and 22 days (IQR:
12-36; maximum: 192) for cases vaccinated with three doses.

\section{Hospitalisation}

Overall, 91 (0.2\%) Omicron and 552 (1.1\%) Delta cases were hospitalised. The median time from positive test to admission was 1 day (IQR: $0-3$ ) for Omicron and 4 days (IQR: 0-7) for Delta.

Using stratified Cox proportional hazard regression, we estimated the risk (adjusted hazard ratio (aHR)) of hospitalisation for infection with the Omicron compared with the Delta variant. The models were stratified by county of residence and sampling date and further adjusted for age group, sex, country of birth, underlying comorbidities and vaccination status. In this main analysis, Omicron was associated with an overall $73 \%$ reduced risk of hospitalisation $(\mathrm{aHR}=0.27$; 95\% confidence interval (Cl): 0.20-0.36) compared with Delta (Table 2). In section 3 of the Supplement we present detailed information on the statistical analysis and hazard ratio estimates for hospitalisation from univariate and multivariable Cox regression for this analysis. 
TABLE 1

Distribution of COVID-19 cases in the retrospective study cohort, by detected variant, for different characteristics and proportion hospitalised, Norway, 6 December 2021-9 January $2022(\mathrm{n}=91,005)$

\begin{tabular}{|c|c|c|c|c|c|c|c|c|c|c|c|c|}
\hline \multirow{3}{*}{ Characteristic } & \multirow{2}{*}{\multicolumn{2}{|c|}{ Study cohort }} & \multicolumn{4}{|c|}{$\begin{array}{l}\text { Variant type } \\
\text { (\% by characteristic) }\end{array}$} & \multicolumn{6}{|c|}{$\begin{array}{c}\text { Hospitalised cases } \\
\text { (\% of diagnosed cases) }\end{array}$} \\
\hline & & & \multicolumn{2}{|c|}{ Delta } & \multicolumn{2}{|c|}{ Omicron } & \multicolumn{2}{|c|}{ Delta } & \multicolumn{2}{|c|}{ Omicron } & \multicolumn{2}{|c|}{ Total } \\
\hline & $n$ & $\%$ & $\mathrm{n}$ & $\%$ & $\mathrm{n}$ & $\%$ & $\mathrm{n}$ & $\%$ & $\mathrm{n}$ & $\%$ & $n$ & $\%$ \\
\hline Total & 91,005 & 100 & 51,481 & 100 & 39,524 & 100 & 552 & 100 & 91 & 100 & 643 & 100 \\
\hline \multicolumn{13}{|l|}{ Sex } \\
\hline Female & 45,262 & 50 & 25,577 & 50 & 19,685 & 50 & 228 & 41 & 53 & 58 & 281 & 44 \\
\hline Male & 45,743 & 50 & 25,904 & 50 & 19,839 & 50 & 324 & 59 & 38 & 42 & 362 & 56 \\
\hline \multicolumn{13}{|l|}{ Age group (years) } \\
\hline $0-29$ & 45,773 & 50 & 25,606 & 50 & 20,167 & 51 & 36 & 6.5 & 11 & 12 & 47 & 7.3 \\
\hline $30-44$ & 23,752 & 26 & 13,496 & 26 & 10,256 & 26 & 95 & 17 & 14 & 15 & 109 & 17 \\
\hline $45-54$ & 11,656 & 13 & 6,537 & 13 & 5,119 & 13 & 80 & 14 & 18 & 20 & 98 & 15 \\
\hline $55-64$ & 6,334 & 7.0 & 3,623 & 7.0 & 2,711 & 6.9 & 116 & 21 & 17 & 19 & 133 & 21 \\
\hline $65-74$ & 2,368 & 2.6 & 1,549 & 3.0 & 819 & 2.1 & 96 & 17 & 11 & 12 & 107 & 17 \\
\hline$\geq 75$ & 1,122 & 1.2 & 670 & 1.3 & 452 & 1.1 & 129 & 23 & 20 & 22 & 149 & 23 \\
\hline \multicolumn{13}{|l|}{ Country of birth } \\
\hline Norway & 66,488 & 73 & 38,639 & 75 & 27,849 & 70 & 323 & 59 & 52 & 57 & 375 & 58 \\
\hline Overseas & 23,568 & 26 & 12,246 & 24 & 11,322 & 29 & 203 & 37 & 34 & 37 & 237 & 37 \\
\hline Unknown & 949 & 1.0 & 596 & 1.1 & 353 & 0.9 & 26 & 4.7 & 5 & 5.5 & 31 & 4.8 \\
\hline \multicolumn{13}{|l|}{ Risk for severe COVID-19 } \\
\hline No underlying comorbidities & 81,036 & 89 & 45,492 & 88 & 35,544 & 90 & 297 & 54 & 36 & 40 & 333 & 52 \\
\hline Medium-risk comorbidity & 8,667 & 10 & 5,159 & 10 & 3,508 & 8.9 & 145 & 26 & 31 & 34 & 176 & 27 \\
\hline High-risk comorbidity & 1,302 & 0.9 & 830 & 1.6 & 472 & 1.2 & 110 & 20 & 24 & 26 & 134 & 21 \\
\hline \multicolumn{13}{|l|}{ Vaccination status at date of positive test } \\
\hline Not vaccinated & 30,546 & 34 & 22,837 & 44 & 7,709 & 20 & 335 & 61 & 15 & 16 & 350 & 34 \\
\hline One dose $<21$ days before positive test & 517 & 0.6 & 355 & 0.7 & 162 & 0.4 & 8 & 1.5 & 1 & \begin{tabular}{|l|}
1.1 \\
\end{tabular} & 9 & 1.4 \\
\hline $\begin{array}{l}\text { Partially completed primary vaccination } \\
\text { series } \geq 21 \text { days before positive test }{ }^{\mathrm{b}}\end{array}$ & 6,507 & 7.2 & 3,935 & 7.6 & 2,572 & 6.5 & 9 & 1.6 & 3 & $3 \cdot 3$ & 12 & 1.9 \\
\hline $\begin{array}{l}\text { Completed primary vaccination series with } \\
\text { maximum two doses 7-179 days before } \\
\text { positive test }^{\mathrm{b}}\end{array}$ & 39,821 & 44 & 17,981 & 35 & 21,840 & 55 & 47 & 8.5 & 26 & 29 & 73 & 11 \\
\hline $\begin{array}{l}\text { Completed primary vaccination series with } \\
\text { maximum two doses } \geq 180 \text { days before } \\
\text { positive test }^{\mathrm{b}}\end{array}$ & 8,351 & 9.2 & 4,790 & 9.3 & 3,561 & 9.0 & 98 & 18 & 26 & 29 & 124 & 19 \\
\hline $\begin{array}{l}\text { Vaccinated with three doses } \geq 7 \text { days before } \\
\text { positive test }{ }^{b}\end{array}$ & 4,848 & $5 \cdot 3$ & 1,505 & 2.9 & 3,343 & 8.5 & 55 & 10 & 20 & 22 & 75 & 12 \\
\hline $\begin{array}{l}\text { Unvaccinated, but previously diagnosed } \\
\text { with COVID-19 6-12 months before positive } \\
\text { test }\end{array}$ & 415 & 0.5 & 78 & 0.2 & 337 & 0.9 & 0 & 0.0 & 0 & 0.0 & 0 & 0.0 \\
\hline
\end{tabular}

COVID-19: coronavirus disease.

a Risk for severe disease based on underlying comorbidities that are associated with a moderate or high risk of serious illness regardless of age. Details on the definitions used are provided in the Supplement, section 1.

${ }^{b}$ See Supplement section 1 for full details on how each vaccination status category is defined and data on vaccine type. Overall, $98 \%$ of vaccinated Omicron cases and $96 \%$ of Delta cases had received a homologous or mixed regimen of mRNA vaccines. The three-dose category predominantly includes cases who received their third dose as a booster dose, however it will also include cases who received their third dose as part of their primary series, for example those severely immunocompromised [14]. We were not able to clearly distinguish persons who had received a third dose as part of their primary series from those who had received a booster dose. 
In Table 2, we present a subgroup analysis of the risk of hospitalisation following infection with the Omicron compared with the Delta variant. The aHR indicated a similar association (reduced risk), except for strata with a small number of cases and few hospitalisations and for some subgroups by vaccination status. We observed interactions between vaccination status and (i) variant, (ii) age group and (iii) underlying comorbidities. We chose not to include these interactions in the main analysis, and investigated them separately in a subgroup analysis by vaccination status (Table 3 ). In this subgroup analysis, the reduction in the risk of hospitalisation for Omicron compared with Delta was smaller among cases who had completed primary vaccination with maximum two doses 7-179 days before positive test compared with unvaccinated cases $(66 \%$ for Omicron vs 93\% for Delta with $\mathrm{Cl}$ that did not overlap). For those vaccinated with three doses, the reduction in the risk of hospitalisation compared with unvaccinated cases was similar for Omicron and Delta cases ( $86 \%$ and $88 \%$ respectively with overlapping $\mathrm{Cl}$ ). However, among Omicron cases, we did not observe a significant decrease in risk for persons who had partially completed the primary vaccination series, nor persons who had completed primary vaccination with maximum two doses $\geq 180$ days before positive test compared with unvaccinated.

In Figure 2, we present the 10-day average case hospitalisation rate (unadjusted) over time. The decrease in the rate in the early part of the plot corresponds to the ongoing roll-out of third doses, either as part of the primary series for persons with specific immunosuppressive conditions (from September 2021), or a booster dose (first offered to persons $\geq 65$ years and care home residents from October 2021). We see a further decrease during late December and early January when the Omicron variant superseded Delta. This latter decrease is around $70 \%$, indicating a population-level effect similar to the decrease observed in the study cohort.

\section{Length of stay in hospital and intensive care admission among hospitalised cases}

At the end of follow-up, 10 of 91 (11\%) patients infected with the Omicron variant and 80 of 552 (14\%) patients infected with the Delta variant were still hospitalised. The crude median LoS among Omicron patients was 2.8 days (IQR: $1.6-6.8$ ) compared with 6.5 (IQR: 3.212.3) among Delta patients. Seven Omicron patients (7.7\%) were admitted to an ICU, compared with 135 (24\%) Delta patients.

The aHR for discharge from hospital and risk of ICU admission for Omicron patients compared with Delta patients were calculated using Cox proportional hazard regression stratified for age, sex, vaccination status and number of underlying risk factors (see Supplement section 3 for detailed information on the statistical analysis). The aHR for discharge for Omicron patients compared with Delta patients was 1.44 (95\% Cl:
0.99-2.07). Assuming exponential distribution of the survival data, an aHR of 1.44 represents an expected $31 \%$ shorter LoS (95\% Cl: $1 \%$ longer-52\% shorter). See Supplement section 4 for the fit of the LoS outcome to an exponential distribution. The aHR for the risk of ICU admission for Omicron patients compared with Delta patients was 0.51 (95\% Cl: 0.20-1.29).

\section{Reported deaths}

Ten (seven non-hospitalised) and 92 (30 non-hospitalised) deaths were reported among the 39,524 Omicron and 51,481 Delta cases, respectively. Nine Omicron and 80 Delta deaths were reported as COVID-19 related (see Supplement section 1 for the definition of a COVID19-related death). We did not further analyse data on deaths because the numbers were small.

\section{Ethical statement}

Ethical approval was granted by Regional Committees for Medical and Health Research Ethics South East (reference number 249509). The need for informed consent was waived.

\section{Discussion}

In this national register-based study, we found that reported COVID-19 cases infected with SARS-CoV-2 Omicron (subvariant BA.1) were associated with a $73 \%$ lower risk of hospitalisation compared with reported infection with the Delta variant. Our findings add to the growing evidence that people infected with Omicron have a lower risk of severe disease than those infected with Delta. Results presented in national reports from Denmark and the United Kingdom (UK), and studies from the United States and Canada estimated a $36-66 \%$ reduced risk of hospitalisation [5-8]. Our preliminary data on LoS and risk of ICU admission also indicate a probable milder disease trajectory among hospitalised Omicron patients than Delta patients, as reported by others [7]. However, our analysis for LoS and risk of ICU admission is based on a small cohort of hospitalised Omicron patients. Results for these outcomes and must be interpreted with caution at this early stage, and will require further investigation in larger patient cohorts.

We detected an interaction between variant and vaccination status. In the subgroup analysis, estimates for cases who had completed primary vaccination with maximum two doses 7-179 days indicated a lower protective effect of the vaccine for Omicron cases compared with Delta (66\% and 93\%, respectively). For other groups vaccinated with one or two doses, the estimates among Omicron cases were too uncertain to draw clear conclusions. The third dose of the vaccine was associated with a similar reduction in the risk of hospitalisation for Omicron and Delta compared with their respective unvaccinated cases $(86 \%$ and $88 \%$, respectively). These findings concur with reports from UK [6] and are expected, based on data from studies that estimated reduced vaccine effectiveness against 
TABLE 2

Hazard ratio estimates for hospitalisation with the Omicron compared with the Delta variant of SARS-CoV-2, using stratified Cox regression, overall and by subgroup analysis, Norway, 6 December 2021-9 January 2022 (n = 91,005)

\begin{tabular}{|c|c|c|c|c|c|}
\hline & \multicolumn{5}{|c|}{ Hospitalisation } \\
\hline & \multicolumn{2}{|c|}{ Delta cases } & \multicolumn{2}{|c|}{$\begin{array}{l}\text { Omicron } \\
\text { cases }\end{array}$} & \multirow{2}{*}{$\begin{array}{l}\text { Omicron vs Delta, adjusted } \\
\text { hazard ratio }(95 \% \mathrm{Cl})\end{array}$} \\
\hline & $\mathrm{n}$ & $\%^{a}$ & $n$ & $\%^{a}$ & \\
\hline Overall/main analysis & 552 & 1.1 & 91 & 0.2 & $0.27(0.20-0.36)$ \\
\hline \multicolumn{6}{|l|}{ Subgroup analysis by } \\
\hline \multicolumn{6}{|l|}{ Sex } \\
\hline Female & 228 & 0.9 & 53 & 0.3 & $0.45(0.30-0.67)$ \\
\hline Male & 324 & 1.3 & 38 & 0.2 & $0.17(0.11-0.27)$ \\
\hline \multicolumn{6}{|l|}{ Age group (years) } \\
\hline $0-29$ & 36 & 0.1 & 11 & 0.1 & $0.24(0.09-0.60)$ \\
\hline $30-44$ & 95 & 0.7 & 14 & 0.1 & $0.23(0.11-0.47)$ \\
\hline $45-54$ & 80 & 1.2 & 18 & 0.4 & $0.40(0.19-0.85)$ \\
\hline $55-64$ & 116 & 3.2 & 17 & 0.6 & $0.22(0.10-0.45)$ \\
\hline $65-74$ & 96 & 6.2 & 11 & 1.3 & $0.20(0.07-0.51)$ \\
\hline$\geq 75$ & 129 & 19 & 20 & 4.4 & $0.41(0.17-0.98)$ \\
\hline \multicolumn{6}{|l|}{ Country of birth } \\
\hline Norway & 323 & 0.8 & 52 & 0.2 & $0.27(0.18-0.40)$ \\
\hline Overseas & 203 & 1.7 & 34 & 0.3 & $0.23(0.14-0.38)$ \\
\hline Unknown & 26 & 4.4 & 5 & 1.4 & $2.33(0.13-41.6)$ \\
\hline \multicolumn{6}{|l|}{ Risk for severe COVID-19 ${ }^{b}$} \\
\hline No underlying comorbidities & 297 & 0.7 & 36 & 0.1 & $0.23(0.15-0.36)$ \\
\hline Medium-risk comorbidity & 145 & 2.8 & 31 & 0.9 & $0.35(0.21-0.59)$ \\
\hline High-risk comorbidity & 110 & 13 & 24 & 5.1 & $0.16(0.06-0.42)$ \\
\hline \multicolumn{6}{|l|}{ Vaccination status at date of positive test } \\
\hline Not vaccinated & 335 & 1.5 & 15 & 0.2 & $0.13(0.07-0.23)$ \\
\hline One dose $<21$ days before positive test & 8 & 2.3 & 1 & 0.6 & NA \\
\hline $\begin{array}{l}\text { Partially completed primary vaccination series } \geq 21 \text { days before positive } \\
\text { test }^{c}\end{array}$ & 9 & 0.2 & 3 & 0.1 & NA \\
\hline $\begin{array}{l}\text { Completed primary vaccination series with maximum two doses 7-179 days } \\
\text { before positive test }{ }^{c, d}\end{array}$ & 47 & 0.3 & 26 & 0.1 & $0.62(0.31-1.24)$ \\
\hline $\begin{array}{l}\text { Completed primary vaccination series with maximum two doses } \geq 180 \text { days } \\
\text { before positive test }{ }^{c, d}\end{array}$ & 98 & 2.1 & 26 & 0.7 & $0.50(0.25-1.01)$ \\
\hline Vaccinated with three doses $\geq 7$ days before positive test ${ }^{c}$ & 55 & 3.7 & 20 & 0.6 & $0.19(0.08-0.43)$ \\
\hline $\begin{array}{l}\text { Unvaccinated, but previously diagnosed with COVID-19 6-12 months before } \\
\text { positive test }\end{array}$ & o & 0.0 & 0 & 0.0 & $\mathrm{NA}^{\mathrm{e}}$ \\
\hline
\end{tabular}

$\mathrm{Cl}$ : confidence interval; COVID-19: coronavirus disease; NA: not available due to small numbers and lack of discordant pairs; SARS-CoV-2: severe acute respiratory syndrome coronavirus 2 .

a Proportions here are calculated using as denominator the respective population in the subgroup and variant as reported in Table 1.

${ }^{b}$ Risk for severe disease based on underlying comorbidities that are associated with a moderate or high risk of serious illness regardless of age. Details on the definitions used are provided in Supplement section 1.

c See Supplement section 1 for full details on how each vaccination status category is defined and data on vaccine type. Overall, $98 \%$ of vaccinated Omicron cases and $96 \%$ of Delta cases had received a homologous or mixed regimen of mRNA vaccines. The three-dose category predominantly includes cases who received their third dose as a booster dose, however it will also include cases who received their third dose as part of their primary series, for example those severely immunocompromised [14]. We were not able to clearly distinguish persons who had received a third dose as part of their primary series from those who had received a booster dose.

d When we used a recoded variable for vaccination status including both categories for those who had completed primary vaccination with maximum two doses, regardless of the time since last dose, we found an adjusted hazard ratio of 0.54 ( $95 \% \mathrm{Cl}: 0.34-0.87$ ).

e We had 415 cases who were unvaccinated, but who had previously been diagnosed with COVID-19 6-12 months before positive test. None of these 415 required hospitalisation. This indicates that prior infection is associated with lower risk of hospitalisation than unvaccinated status, but we could not calculate estimates because of a lack of discordant pairs in our model.

Hazard ratios were estimated using Cox regression stratified by county of residence and date of sampling and further adjusted for variant, sex, age group, country of birth, underlying comorbidities and vaccination status at date of positive test. 
Subgroup analysis using stratified Cox regression for the risk of hospitalisation by vaccination status among COVID-19 cases, Norway, 6 December 2021-9 January $2022(\mathrm{n}=91,005)$

\begin{tabular}{|c|c|c|c|c|c|c|}
\hline & \multicolumn{6}{|c|}{ Adjusted hazard ratio $(95 \% \mathrm{Cl})$ for hospitalisation } \\
\hline & $\begin{array}{c}\text { Not } \\
\text { vaccinated }\end{array}$ & $\begin{array}{l}\text { One dose }<21 \\
\text { days before } \\
\text { positive test }\end{array}$ & $\begin{array}{c}\text { Partially } \\
\text { completed } \\
\text { primary } \\
\text { vaccination } \\
\text { series } \geq 21 \text { days } \\
\text { before positive } \\
\text { test }^{\mathrm{a}}\end{array}$ & $\begin{array}{c}\text { Completed primary } \\
\text { vaccination series } \\
\text { with maximum two } \\
\text { doses } 7-179 \text { days } \\
\text { before positive }^{\text {test }}\end{array}$ & $\begin{array}{c}\text { Completed } \\
\text { primary } \\
\text { vaccination series } \\
\text { with maximum } \\
\text { two doses } \geq 180 \\
\text { days before } \\
\text { positive test }{ }^{\mathrm{a}, \mathrm{b}}\end{array}$ & $\begin{array}{c}\text { Vaccinated with } \\
\text { three doses } \geq 7 \\
\text { days before } \\
\text { positive test }^{a}\end{array}$ \\
\hline Overall/main analysis & Ref & $1.18(0.59-2.39)$ & $0.22(0.12-0.41)$ & $0.09(0.07-0.12)$ & $0.17(0.14-0.22)$ & $0.10(0.07-0.14)$ \\
\hline \multicolumn{7}{|l|}{ Subgroup analysis by } \\
\hline \multicolumn{7}{|l|}{ Variant } \\
\hline Delta & Ref & $1.18(0.56-2.50)$ & $0.19(0.09-0.38)$ & $0.07(0.05-0.10)$ & $0.16(0.12-0.21)$ & $0.12(0.09-0.17)$ \\
\hline Omicron & Ref & $2.03(0.24-17.4)$ & $0.71(0.20-2.51)$ & $0.34(0.17-0.68)$ & $0.59(0.28-1.22)$ & $0.14(0.06-0.31)$ \\
\hline \multicolumn{7}{|l|}{ Age group (years) } \\
\hline $0-29$ & Ref & $5.72(1.26-26.1)$ & $0.16(0.02-1.16)$ & $0.45(0.19-1.08)$ & $1.53(0.47-4.94)$ & $0.57(0.05-6.63)$ \\
\hline $30-44$ & Ref & NA & $0.20(0.05-0.84)$ & $0.07(0.04-0.13)$ & $0.27(0.13-0.55)$ & $0.29(0.10-0.84)$ \\
\hline $45-54$ & Ref & $1.33(0.28-6.28)$ & $0.33(0.08-1.42)$ & $0.09(0.05-0.16)$ & $0.15(0.07-0.31)$ & $0.26(0.11-0.60)$ \\
\hline $55-64$ & Ref & $1.50(0.15-14.5)$ & $0.16(0.02-1.22)$ & $0.05(0.03-0.10)$ & $0.12(0.07-0.20)$ & $0.17(0.08-0.34)$ \\
\hline $65-74$ & Ref & $2.36(0.31-18.2)$ & $0.54(0.11-2.74)$ & $0.17(0.07-0.42)$ & $0.11(0.06-0.21)$ & $0.09(0.04-0.18)$ \\
\hline$\geq 75$ & Ref & $3.27(0.41-25.8)$ & $0.40(0.08-1.88)$ & $0.29(0.10-0.83)$ & $0.27(0.15-0.50)$ & $0.04(0.02-0.09)$ \\
\hline \multicolumn{7}{|l|}{ Risk for severe COVID-19 } \\
\hline $\begin{array}{l}\text { No underlying } \\
\text { comorbidities }\end{array}$ & Ref & $1.11(0.50-2.44)$ & $0.28(0.14-0.58)$ & $0.06(0.04-0.09)$ & $0.13(0.08-0.19)$ & $0.03(0.01-0.07)$ \\
\hline Medium-risk comorbidity & Ref & $\begin{array}{c}0.64 \\
(0.08-5.05) \\
\end{array}$ & $0.18(0.04-0.79)$ & $0.16(0.10-0.26)$ & $0.20(0.14-0.30)$ & $0.07(0.04-0.12)$ \\
\hline High-risk comorbidity & Ref & $\begin{array}{c}7.96 \\
(0.41-155.7) \\
\end{array}$ & $1.53(0.16-14.4)$ & $0.29(0.08-1.11)$ & $0.87(0.36-2.08)$ & $0.63(0.26-1.56)$ \\
\hline
\end{tabular}

aHR: adjusted hazard ratio; CI: confidence interval; NA: not available due to small numbers and lack of lack of discordant pairs; COVID-19: coronavirus disease.

a See Supplement section 1 for full details on how each vaccination status category is defined and data on vaccine type. Overall, $98 \%$ of vaccinated Omicron cases and $96 \%$ of Delta cases had received a homologous or mixed regimen of mRNA vaccines. The three-dose category predominantly includes cases who received their third dose as a booster dose, however it will also include cases who received their third dose as part of their primary series, for example those severely immunocompromised [14]. We were not able to clearly distinguish persons who had received a third dose as part of their primary series from those who had received a booster dose.

${ }^{b}$ When we used a recoded variable for vaccination status including both categories for those who had completed primary vaccination with maximum two doses, regardless of the time since last dose, we estimated that Omicron cases had a $50 \%$ reduced risk (aHR $=0.50 ; 95 \%$ $\mathrm{Cl}: 0.26-0.96)$ and Delta cases an $88 \%$ reduced risk of hospitalisation (aHR $=0.12 ; 95 \% \mathrm{Cl}: 0.09-0.14$ ), respectively, compared with unvaccinated.

' Risk for severe disease based on underlying comorbidities that are associated with a moderate or high risk of serious illness regardless of age. Details on the definitions of medium- and high-risk categories are provided in Supplement section 1.

Hazard ratios for hospitalisation were estimated using Cox regression stratified by county of residence and date of sampling and further adjusted for variant, sex, age group, country of birth and underlying comorbidities at date of positive test.

Omicron infection [9-12] and the benefit of a booster dose $[9,10]$.

During the study period, hospitals functioned within capacity, and the testing strategy in Norway was stable, with a high proportion of reported cases screened (59\%) for variant. We stratified by sampling date and county of residence, considering differences in testing and screening by time and place. Although we noticed a slightly higher proportion of hospitalised cases being screened compared with non-hospitalised, we believe that this bias is limited. If we assume that we have oversampled hospitalised Delta cases, given the estimated higher risk of hospitalisation, this would cause us to slightly overestimate the reduction in the risk of hospitalisation for Omicron. Conversely, another potential bias could be systematic differences between the variants among non-diagnosed cases. For example, Omicron infections may have a higher rate of asymptomatic carriage than other variants of concern [13]. Such infections may be less likely to be diagnosed, which could result in an underestimation of the true reduction in risk of hospitalisation for Omicron compared with Delta.

\section{Conclusion}

Information on severity of infection with new variants is central for decision-making on control measures and strategies. However, considering the increased transmissibility of the Omicron variant and the reduced 


\section{FIGURE 2}

10-day average case COVID-19 hospitalisation rate (unadjusted) as a function of time, Norway, 1 November 2021- 9 January $2022(\mathrm{n}=239,391)$

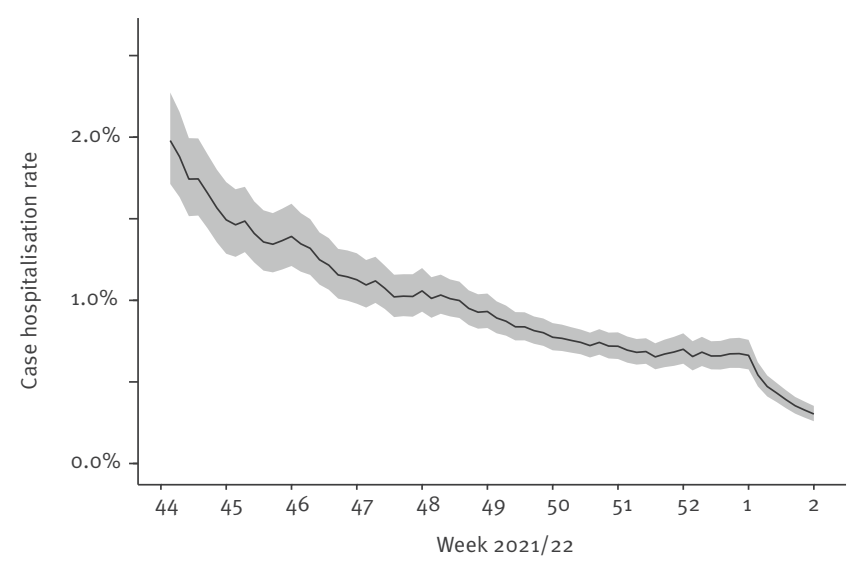

The figure shows the case hospitalisation rate by date of sampling. This rate is calculated as the 10-day averaged fraction of patients who are admitted to hospital (10-day average of hospitalised cases/reported cases by date of sampling). The calculation of case hospitalisation rate discounts the incidence of cases during the latest dates by the observed delay between testing and admission to account for potential right censoring [15].

vaccine effectiveness against Omicron infection, we need to consider that the lower risk of severe disease associated with Omicron does not necessarily imply a reduced burden on hospitals, especially in the event of large waves of infections during the winter season when other viruses also circulate.

\section{Acknowledgements}

First and foremost, we wish to thank all those who have helped report data to the national emergency preparedness registry at the Norwegian Institute of Public Health (NIPH) throughout the pandemic. We also highly acknowledge the efforts that regional laboratories have put into establishing a routine variant screening procedure or whole genome sequencing at short notice and registration of all analysis in national registries for surveillance. Thanks also to the staff at the Virology and Bacteriology departments at NIPH involved in national variant identification and whole genome analysis of SARS-CoV-2 viruses. We also highly acknowledge the efforts of staff at hospitals around Norway to ensure the reporting of timely and complete data to the Norwegian Intensive Care and Pandemic Registry, as well as colleagues at the register itself. We would also like to thank Anja Elsrud Schou Lindman, project director for the national preparedness registry, and all those who have enabled data transfer to this registry, especially Gutorm Høgåsen at the NIPH, who has been in charge of the establishment and administration of the registry. We would also like to thank 'Team risk group' at the NIPH, who developed the data cleaning procedure for underlying comorbidities in the preparedness registry.

Funding: No specific funding received. The study was performed as part of routine work at the Norwegian Institute of Public Health.

\section{Conflict of interest}

None declared.
Authors' contributions

LaVe, HB, ABK, JeS, KB, GR, LiVo, KN, EB and RW were involved in the conceptualisation of the study. RW drafted the study protocol, with input from LaVe, HB, ABK, MS, ES, KN and $\mathrm{EB}$, and coordinated the study. $\mathrm{OH}, \mathrm{MS}, \mathrm{NA}, \mathrm{RK}, \mathrm{KB}$ and $E A B$ contributed directly to the acquisition of data. LaVe, HB, JeS, OH, MS, NA, JoS, ES, KB and RW contributed to data cleaning, verification and/or preparation. LaVe, HB, ABK, JeS, JoS, ES and RW had access to the final linked dataset. LaVe conducted the statistical analysis for risk of hospitalisation and ABK for length of hospital stay and ICU admission in consultation with HB, JeS, JoS, GR and RW. All co-authors contributed to the interpretation of the results. LaVe, HB and RW drafted the manuscript. All co-authors contributed to the revision of the manuscript and approved the final version for submission.

\section{References}

1. Brandal LT, MacDonald E, Veneti L, Ravlo T, Lange H, Naseer $\mathrm{U}$, et al. Outbreak caused by the SARS-CoV-2 Omicron variant in Norway, November to December 2021. Euro Surveill. 2021;26(50):2101147. https://doi.org/10.2807/1560-7917. ES.2021.26.50.2101147 PMID: 34915975

2. Norwegian Institute of Public Health (FHI). Ukerapport uke 2 (10.01 - 16.01.22): Ukerapporter om koronavirus og covid-19. [Weekly report week 2 (10-16 Jan 2022): Weekly reports about coronavirus and COVID-19]. Oslo: FHI; 2022. Norwegian. Available from: https://www.fhi.no/contentassets/8a971e 7boa3c4ao6bdbf381ab52e6157/vedlegg/2022/ukerapportuke-2-10.01---16.01.22.pdf

3. Norwegian Institute of Public Health (FHI). Ukerapport uke 52 (27.12.21 - 02.01.22): Ukerapporter om koronavirus og covid-19. [Weekly report week 52 (27 Dec 2021-2 Jan 2022): Weekly reports about coronavirus and COVID-19]. Oslo: FHI; 2022. Norwegian. Available from: https://www.fhi.no/content assets/8a971e7boa3c4a06bdbf381ab52e6157/vedlegg/2021/ ukerapport-uke-52-27.12.21---02.01.22.pdf

4. Norwegian Institute of Public Health (FHI). Emergency preparedness register for COVID-19 (Beredt C19). Oslo: FHI; 2021. Available from: https://www. fhi.no/en/id/infectious-diseases/coronavirus/ emergency-preparedness-register-for-covid-19

5. Statens Serum Institut (SSI). Status of the SARS-CoV-2 variant Omicron in Denmark. Copenhagen: SSI; 2022. Available from: https://files.ssi.dk/covid19/omikron/statusrapport/ rapport-omikronvarianten-03012022-9gj3

6. UK Health Security Agency. SARS-CoV-2 variants of concern and variants under investigation in England. Technical briefing: Update on hospitalisation and vaccine effectiveness for Omicron VOC-21NOV-01 (B.1.1.529). London: UK Health Security Agency; 2021. Available from: https://assets.publishing. service.gov.uk/government/uploads/system/uploads/ attachment_data/file/1044481/Technical-Briefing-31-Dec2021-Omicron_severity_update.pdf

7. Lewnard JA, Hong VX, Patel MM, Kahn R, Lipsitch M, Tartof SY. Clinical outcomes among patients infected with Omicron (B.1.1.529) SARS-CoV-2 variant in southern California. medRxiv.2022.01.11.22269045. preprint. https://doi.org/ https://doi.org/10.1101/2022.01.11.22269045 https://doi.org/1 $0.1101 / 2022.01 .11 .22269045$

8. Ulloa AC, Buchan SA, Daneman N, Brown KA. Early estimates of SARS-CoV-2 Omicron variant severity based on a matched cohort study, Ontario, Canada. medRxiv.2021.12.24.21268382. preprint. https://doi.org/ https://doi.org/10.1101/2021.12.24. 21268382

9. Andrews N, Stowe J, Kirsebom F, Toffa S, Rickeard T, Gallagher E, et al. Effectiveness of COVID-19 vaccines against the Omicron (B.1.1.529) variant of concern. medRxiv.2021.12.14.21267615. preprint. https://doi.org/ https://doi.org/10.1101/2021.12.14.21267615 https://doi.org/1 $0.1101 / 2021.12 .14 .21267615$

10. Buchan SA, Chung H, Brown KA, Austin PC, Fell DB, Gubbay $J B$, et al. Effectiveness of COVID-19 vaccines against Omicron or Delta infection. medRxiv,2021.12.30.21268565. preprint. https://doi.org/ https://doi.org/10.1101/2021.12.30.21268565 https://doi.org/10.1101/2021.12.30.21268565

11. Lyngse FP, Hvas Mortensen L, Denwood MJ, Engbo Christiansen L, Holten Møller C, Skov RL, et al. SARS-CoV-2 Omicron VOC transmission in Danish households. medRxiv. 2021.12.27.21268278. preprint. https://doi.org/ https://doi.or 
g/10.1101/2021.12.27.21268278 https://doi.org/10.1101/2021. 12.27.21268278

12. Hansen $\mathrm{CH}$, Blicher Schelde A, Rask Moustsen-Helm I, Emborg H-D, Grove Krause T, Mølbak K, et al. Vaccine effectiveness against SARS-CoV-2 infection with the Omicron or Delta variants following a two-dose or booster BNT162 b2 or mRNA-1273 vaccination series: A Danish cohort study. medRxiv.2021.12.20.21267966. preprint. https://doi.org/ https://doi.org/10.1101/2021.12.20.21267966 https://doi.org/1 $0.1101 / 2021.12 .20 .21267966$

13. Garrett N, Tapley A, Andriesen J, Seocharan I, Fisher LH, Bunts L, et al. High rate of asymptomatic carriage associated with variant strain Omicron. medRxiv.2021.12.20.21268130. preprint. https://doi.org/ https://doi.org/10.1101/2021.12.20.2 1268130 https://doi.org/10.1101/2021.12.20.21268130

14. Folkehelseinstituttet (FHI). Flere med alvorlig nedsatt immunforsvar bør ta 3 . dose koronavaksine. [More people with a severely impaired immune system should take a 3 rd dose of corona vaccine]. Oslo: FHI; 2021. Norwegian Available from: https://www.fhi.no/nyheter/2021/flere-med-alvorlig-nedsattimmunforsvar-bor-ta-3.-dose-koronavaksine

15. Nishiura H, Klinkenberg D, Roberts M, Heesterbeek JA. Early epidemiological assessment of the virulence of emerging infectious diseases: a case study of an influenza pandemic. PLoS One. 2009;4(8):e6852. https://doi.org/10.1371/journal. pone.0006852 PMID: 19718434

\section{License, supplementary material and copyright}

This is an open-access article distributed under the terms of the Creative Commons Attribution (CC BY 4.0) Licence. You may share and adapt the material, but must give appropriate credit to the source, provide a link to the licence and indicate if changes were made.

Any supplementary material referenced in the article can be found in the online version.

This article is copyright of the authors or their affiliated institutions, 2022. 